\title{
Ouarsenis : Histoire, géographie humaine et société
}

Jean-Pierre Laporte, Salem Chaker, Colette Roubet et Jacques Vignet-

Zunz

\section{OpenEdition}

Journals

Édition électronique

URL : https://journals.openedition.org/encyclopedieberbere/2853

DOI : 10.4000/encyclopedieberbere.2853

ISSN : 2262-7197

\section{Éditeur}

Peeters Publishers

\section{Édition imprimée}

Date de publication : 2 juin 2013

Pagination : 5929-5945

ISBN : 978-2-7584-0194-0

ISSN : 1015-7344

\section{Référence électronique}

Jean-Pierre Laporte, Salem Chaker, Colette Roubet et Jacques Vignet-Zunz, « Ouarsenis : Histoire, géographie humaine et société », Encyclopédie berbère [En ligne], 36 | 2013, document O39, mis en ligne le 12 mars 2021, consulté le 17 février 2022. URL : http://journals.openedition.org/ encyclopedieberbere/2853; DOI : https://doi.org/10.4000/encyclopedieberbere.2853

Ce document a été généré automatiquement le 17 février 2022.

(c) Tous droits réservés 


\section{Ouarsenis : Histoire, géographie humaine et société}

Jean-Pierre Laporte, Salem Chaker, Colette Roubet et Jacques Vignet-

Zunz

\section{Limites géographiques}

1 Pour les historiens médiévaux et les géographes modernes, le massif s'étend sur environ $200 \mathrm{~km}$ d'est en ouest, tandis que sa largeur est de l'ordre de $100 \mathrm{~km}$, sauf à l'est, où elle ne dépasse pas $60 \mathrm{~km}$ à la hauteur du méridien de Derrag. Il s'inscrit entre le fleuve Chélif et la ville de Chlef (ex-El-Asnam, ex-Orléanville) au nord, le Titteri et Médéa à l'est, la rivière Nahr Ouassal, le plateau du Sersou et Tiaret au sud, l'oued Mina, les monts de Saïda et la région de Relizane (Ighil Izane) à l'ouest. Les habitants semblent englober sous ce nom un périmètre plus restreint, plus ou moins réduit à la partie centrale, en excluant les parties orientale et occidentale.

2 Pendant la période coloniale française, le massif faisait partie du département d'Alger ; actuellement, il est à cheval sur les wilayas de Médéa, Aïn Defla (ex-Duperré), Tissemsilt (ex-Vialar), Chlef (Orléanville, puis El-Asnam), Relizane et Tiaret. 


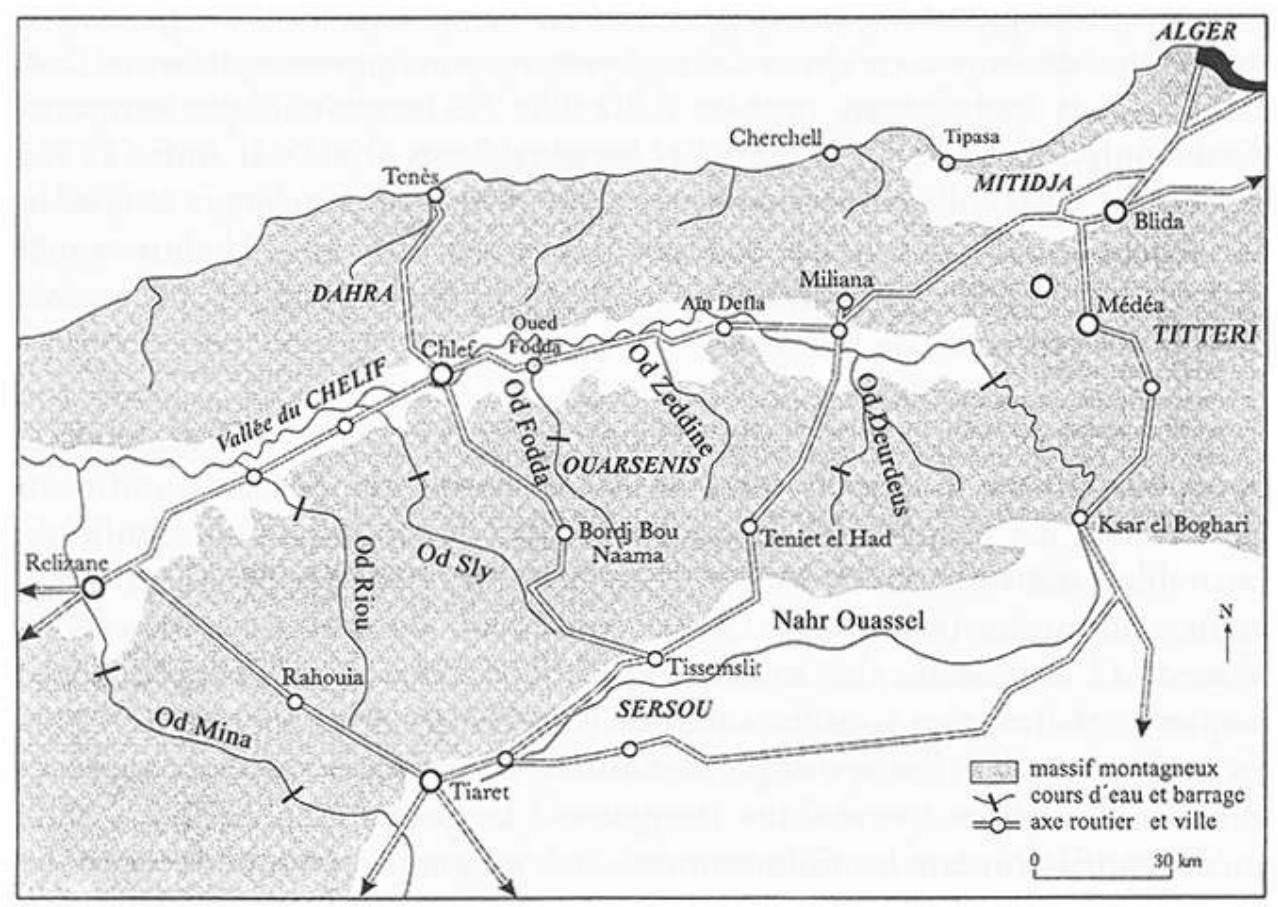

Fig. 1 : LE RELIEF, LES FLEUVES, LES BARRAgES ET LES VILLES DE L'OUARSENIS, D'APRÈS M. CôTE, E.I., T. XI, 2005, P. 152, ICI COMPLÉTÉ.

\section{Relief et géographie}

3 L'Ouarsenis fait partie intégrante de l'Atlas tellien* occupant l'Algérie occidentale. Distinct des lignes de hauteurs formées au nord par les sahels collinaires d'Oran et d'Arzeu et les petits chaînons montagneux du Dahra et du Zaccar, l'Ouarsenis constitue avec les Traras et les monts du Tessala, la seconde ligne de crêtes méridionales telliennes, surplombant les hautes plaines intérieures de Marnia, Tlemcen et du Sersou.

Le massif est formé de plissements de schistes, flysch et marnes entassés que dominent quelques vigoureux pointements de calcaire infra-crétacés et jurassiques basculés, notamment le grand dôme calcaire du Kef Sidi Amar, près de Bordj Bou Naama. Le massif, contourné à l'ouest par l'oued Mina, est compartimenté par des affluents du Chélif, qui se dirigent globalement vers le nord/nord-ouest notamment, d'ouest en est, l'oued Rhiou, l'oued Sly, l'oued Fodda, l'oued Zeddine et l'oued Deurdeuss.

5 Le pic Sidi Amar, sommet principal de l'Ouarsenis $(1985 \mathrm{~m})$, domine de $800 \mathrm{~m}$ les reliefs environnants. Il constitue un gigantesque pivot en forme de cône surbaissé à flancs escarpés. On ne sait pas trop à quoi raccorder le surnom d'« Æil du Monde » que les traités scientifiques et les grands atlas du XIX siècle citent comme «donné par les indigènes ", mais qui ne paraît pas avoir d'équivalent dans les parlers locaux. Au nordest, le Sra Abdelkader dresse une imposante muraille calcaire, une sorte de sierra courbée en un grand arc concave vers le sud, long de $5 \mathrm{~km}$ et large d'environ $1 \mathrm{~km}$, découpée en dents de scie comme une gigantesque lame (Sari 1977). Deux qubub (sing. $q u b b a$, sanctuaire de forme cubique surmonté d'une coupole) coiffent les sommets les plus élevés (Kef Sidi Amar et Kef Sidi Abdelqader) ; l'attribution de noms de saints aux principaux sommets, les visites rituelles et repas communiels qui y ont lieu chaque 
année témoignent du rôle qu'ont joué de tout temps les sanctuaires de sommet dans la vie religieuse de la région.

À l'exception de la zone culminante qui se caractérise par de longs et rigoureux hivers, la région offre dans l'ensemble de bonnes conditions climatiques. La plupart des terroirs renferme des sols épais et équilibrés, favorables aux cultures et au couvert forestier. Les précipitations largement suffisantes (cf. notice 038) expliquent l'importance des débits d'oueds et le nombre des sources. Les effets des irrégularités pluviométriques et thermiques sont ici atténués. L'humidité de la montagne assure les moissons et les récoltes, et permet aussi des pâturages réguliers, contrairement à ce qui se passe dans les plaines. Le piémont méridional reste encore suffisamment humide, comme le souligne nettement la vocation céréalière du Sersou. Ainsi, grâce à sa situation géographique, l'Ouarsenis échappe aux influences du sud, même s'il est en contact direct avec le domaine semi-aride.

\section{Histoire}

7 Vaste région naturelle, l'Ouarsenis a rarement été traité par les historiens comme un ensemble en soi, mais comme une annexe plus ou moins rattachée à telle ou telle ville de plaine du voisinage. Ceci réduit par trop ses particularités. Les conquérants successifs se sont peu risqués à l'intérieur, en se contentant d'occuper les piémonts limitrophes, de contrôler les parties céréalières et d'investir des chefs autochtones chargés d'encadrer les tribus et de recouvrer les impôts. L'on ne saurait cependant soutenir une opposition trop forte entre les montagnards et les paysans des plaines. Les populations ne se sont jamais cantonnées étroitement dans les sites défensifs qu'offrent naturellement les reliefs. Pour compléter les ressources de leurs terroirs accidentés, elles avaient besoin aussi de terres de parcours et de cultures, et du concours des populations voisines, aussi bien en périodes de guerre qu'en périodes de paix. On manque d'une synthèse historique qui traiterait des différents aspects de l'Ouarsenis : lieu de refuge, certes, mais aussi lieu de vie en liaison avec les alentours, du fait de la domination des conquérants, mais aussi par des échanges commerciaux, les productions des plaines étant complémentaires de celles des montagnes et inversement.

\section{Préhistoire et protohistoire}

8 Si l'intérieur du massif, insuffisamment exploré, paraît encore aujourd'hui ne pas avoir connu un peuplement préhistorique bien identifié, notamment durant l'Holocène (10 000 BC), on doit cependant à F.-E. Roubet (1947), à R. de Bayle des Hermens (1955, 1956, 1963) et à $P$. Cadenat (1963) le signalement en périphérie du massif de stations d'art rupestre se trouvant dans l'aire d'influence de Columnata* et de Tiaret*. D'autres précisions sur cette période préhistorique et son anthropisation seront fournies sous cette dernière entrée.

\section{Antiquité}

9 Le massif central au moins a livré jadis des bazinas* et des tumuli, d'époque en réalité inconnue, qui mériteraient d'être réexaminés. Tout en plus peut-on en conclure qu'il 
était déjà peuplé avant l'Antiquité classique. On peut sans doute accepter l'identification déjà ancienne de l'Ouarsenis avec l'Ancorarius mons de Pline l'Ancien, selon une hypothèse vraisemblable, mais qui reste fragile, comme le pensait Gsell (Atlas, f. 23, $n^{\circ} 1$ ). Après Gsell et Cat, mais aussi Desanges (s.v. Mukeni*), on peut même voir au moins une partie du massif dans les monts Garapha de Ptolémée (IV, 2, 3, Müller, p. 604), mais vu ce que l'on sait maintenant de ce géographe pour l'intérieur du pays (Laporte 2003), il convient de rester prudent sur ces différents points.

Compte tenu de ces incertitudes géographiques, de l'imprécision des géographes antiques, et en l'absence de témoignages épigraphiques précis, il est difficile de nommer les populations du massif pendant l'Antiquité. Desanges (1962, carte 2 et 2010 s.v. Makkourae) est prêt à placer dans la partie orientale, avant le second siècle de notre ère, les Akouensi, les Mukeni et les Makkourae*, mais accompagne cette localisation d'un point d'interrogation, tandis que Maccui et Makhousii auraient occupé le Dahra et les Nakmousii plutôt le Sersou. En revanche, il n'est pas évident de rapprocher, comme cela a été parfois proposé, les Maghrawa*, tribu médiévale du nord de l'Ouarsenis, des Makkourebi de Ptolémée (IV, 2, 5).

On connaît sans doute mieux les voisins du massif, avec peut-être la gens des Mazices* dans le Dahra, au nord du Chélif (Modéran, «Mazices », EB, XXXI, p. 4800-4801). Mais pour R. Rebuffat (à paraître), il pourrait s'agir d'un groupe couvrant une bonne partie de la Maurétanie césarienne occidentale. Les pays situés à l'ouest de l'Ouarsenis semblent avoir été occupés par des Bavares* «occidentaux " (Desanges 1962, p. 47-48; Camps, « Bavares », EB, IX, p. 1395).

C'est dans ce cadre incertain que le massif semble apparaître vers le milieu du premier siècle chez Pline l'Ancien qui célèbre l'Ancorarius mons pour la production de grands blocs de "citre» (thuya) utilisés pour façonner des plateaux de tables monoxyles: Ancorarius mons vocatur citerioris Mauretaniae, qui laudatissimus dedit citrum, iam exhaustus = C'est le mont Ancorarius, dans la Mauritanie intérieure, qui produisait le citre le plus estimé ; il est déjà épuisé (Histoire naturelle, XIII, 95 : Paris, Les Belles Lettres, 1956, p. 48-49). Ce témoignage remonte en réalité à l'époque de la République romaine. Les notables romains, comme César ou Cicéron, raffolaient de ces tables de très grand luxe ; de même, ensuite, les rois de Maurétanie Juba II* et Ptolémée*, au point que les plus gros arbres de jadis avaient hélas été tous coupés, et le gisement épuisé dès avant Pline (milieu du $\mathrm{I}^{\text {er }}$ siècle après J.-C.).

Le massif subit sans doute tôt l'influence romaine après l'annexion de la Maurétanie par Rome (40 ap. J.-C.), plus sans doute par des échanges que par une véritable soumission.

Par la suite, indices et certitudes sont rares. Vers 200 ap. J.-C., l'installation de la nova pratentura* sévérienne dans le Sersou, au sud de l'Ouarsenis, ne changea guère la situation, au-delà d'un probable contrôle renforcé des nomades qui remontaient sans doute déjà haut sur les pentes méridionales du massif. Il faut renoncer à l'idée de $\mathrm{C}$. Courtois d'un retrait de l'occupation romaine vers 280 sur un limes, frontière qui aurait longé l'oued Rhiou, en abandonnant toute la Maurétanie occidentale. Après avoir découvert des milliaires plus tardifs dans le Sersou, P. Salama (1966, p. 1309-1310 et 2005, notamment p. 287-188) a réduit à néant cette hypothèse. L'Ouarsenis restait entièrement à l'intérieur du territoire romain même si l'intensité de son contrôle reste inconnue. 
15 La grande expédition de Maximien contre les Quinquegentanei* de Kabylie (297-298) se traduisit par une remise en ordre de toute la Maurétanie césarienne. Dans l'Ouarsenis comme ailleurs, de nombreux chefs autochtones nommés par Rome prirent les noms de l'empereur, Aurelius ou Maximianus, au début du IV siècle $^{1}$.

16 L'intérieur du massif semble avoir été peu romanisé, dans la mesure où l'on n'y a signalé que peu de ruines "romaines », souvent plus petites que dans le Sersou (Gsell, Atlas, f. 23), et très souvent mal identifiées faute de révision récente. De toute manière, leur technique de construction ne permet aucune conclusion quant à l'ethnie de leurs constructeurs.

17 Une partie au moins des populations du massif a sans doute été christianisée, sans que l'on en ait à ce jour de preuve (contrairement au Sersou, dans lequel les vestiges chrétiens sont nombreux). Aucun évêque attribuable à l'Ouarsenis n'est mentionné lors des conciles de 411 ou de 484 (il est vrai que la Maurétanie césarienne occidentale y était fort peu représentée).

Dans la première moitié $\mathrm{du} \mathrm{IV}^{\mathrm{e}}$ siècle, des notables chrétiens sont attestés à la périphérie du massif. Les premiers reliefs portent les ruines de "châteaux » (ou de «maisons-fortes ») privés, souvent de plan cruciforme, dominant les plaines au loin, ainsi celui de Ksar el-Kaoua (Laporte, à paraître) dans la montagne, entre Ammi Moussa et Chlef. Comme l'a fait remarquer P. Salama, ils correspondent bien à l'ascension sociale des chefs autochtones qui devait aboutir à la mise en place progressive d'une sorte de féodalité. Selon les mots de Salama (1954, p. 229), « la puissance romaine, sous le couvert de son organisation administrative, se trouvait à la merci des seigneurs locaux ».

On le vit bien lors de la révolte de Firmus (vers 370). En 373, Valentinien envoya pour le soumettre Théodose l'Ancien (père du futur empereur de ce nom). La campagne avait commencé dans l'est de la Maurétanie césarienne. Venant de Tigava (Les Attafs, exWattignies, w. Aïn Defla), après avoir saccagé le domaine fortifié dit « de Gaionas », Théodose «s'avança jusqu'à la forteresse de Tingis par le mont Ancorarius et tomba sur les Mazices qui avaient opéré leur concentration » (Ammien Marcellin, 29, 5, 25).

Si le mont Ancorarius est bien l'Ouarsenis, on comprend que Théodose a fait passer son armée par la montagne pour mieux surprendre dans la plaine du Chélif les Mazices, qui furent contraints au ralliement (Ammien Marcellin, 29, 5, 26), tout comme les Musones (Ammien Marcellin, 29, 5, 27), à rapprocher sans doute des Mukeni*

21 Vers 400, comme le montre la Notitia Dignitatum, la défense des plaines romaines était assurée par des limites (pluriel de limes), non pas une défense linéaire, mais l'organisation de la protection de bassins fertiles séparés. Un seul est identifié, le limes Columnatensis, autour de Sidi Hosni (w. de Tiaret), sur les marges sud-ouest de l'Ouarsenis.

22 En 429, le passage rapide des Vandales de Genséric fit voler en éclat la puissance romaine dans tout l'ouest algérien, et plus encore après la prise de Carthage en 439. Pour être tranquille sur ses arrières occidentaux, Genséric fit tout simplement détruire les remparts des villes romaines, qui regrettaient Rome, pour les mettre à la merci des montagnards.

23 Certes, la Maurétanie césarienne fut rendue à l'Empire en 442, mais cette fiction politico-administrative disparut définitivement avec la reprise en main de la province par Genséric en 455. Dans les faits, une bonne partie du territoire maurétanien échappa 
à la domination vandale, et plus encore après 484. Parallèlement, montait en puissance le grand royaume berbéro-romain dont les souverains furent ensevelis dans les Djedars*. On ne sait trop pourquoi, Courtois l'a parfois nommé «royaume de l'Ouarsenis » alors que son centre de gravité était certainement le Sersou, dans la région de Tiaret-Frenda. Il semble avoir couvert une bonne partie de l'ancienne Maurétanie césarienne (cf. Camps, notice D63, «Djedar », EB XVI, 1995). Il s'assura certes probablement très tôt du contrôle du massif. Comme l'a noté Dj. Sari (1971, p.67), un royaume qui aurait englobé la montagne et les Hautes Plaines (le Sersou) aurait constitué un ensemble économique cohérent, en s'appuyant sur la complémentarité des terroirs (mais il s'agit encore d'une hypothèse de travail).

Après avoir vaincu les Vandales en 533, les Byzantins ne réussirent à contrôler que quelques escales à l'est de Caesarea (Cherchel), tandis que l'essentiel de la province leur échappait. Le royaume des djedars pouvait prospérer en paix et l'Ouarsenis également.

\section{Moyen Âge}

Selon Ibn Khaldoun, la région paraît avoir été conquise et islamisée très tôt. Les Berbères de la région sont donnés comme étant "Zénètes » par les historiens arabes qui les rattachent aux Banu Ifren* et aux Maghraoua* (Ibn Khaldoun, t. I). Ces derniers occupaient le nord de l'Ouarsenis, et Ibn Khaldoun leur attribue une indiscutable identité Botr (cf. Modéran 2003, p. 805). Géographes et historiens médiévaux parlent peu de l'Ouarsenis dans la mesure où le compartimentage orographique du massif faisait, et fait toujours, obstacle aux communications est-ouest et renvoyait les voyageurs vers les routes plus aisées du Chélif et du Sersou.

Après l'effondrement de la dynastie rostémide et l'émigration des kharijites vers Ouargla* et le Mzab*, l'Ouarsenis connut des dominations successives.

Après avoir fondé Marrakech en 1060, l'Almoravide Youçof Ibn Tachfin lança plusieurs expéditions vers l'est. Il s'empara tout d'abord de Fès en 1069, puis s'avança vers l'est, occupant Oujda, Tlemcen, Oran, Ténès, la région de l'Ouarsenis, avant d'arriver devant Alger.

En 546 H/1151, l'Almohade 'Abd el-Moumen conquit les monts Ouancherich (Wanšeriš), Meliana, Almeria, Djezaïr des Beni Mezghanna (Alger) et Bougie.

Au XIV ${ }^{e}$ siècle, selon Ibn Khaldoun (I, 248) : « Un débris des Matmata habite aujourd'hui l'Ouarchenis. Il s'y réfugia à l'époque où les Beni-Toudjin, peuple zénatien, lui enleva le pays de Mindas. C'est maintenant une peuplade soumise à l'impôt». Le "pays de Mindas » était constitué des plateaux de Mindas, voisins de l'Ouarsenis et du Ghezoul, montagne du pays de Tahert.

En 712 et 713 de l'Hégire (1312 et 1313), les Abd-el-Wadides s'emparaient successivement d'Alger et de Dellys, puis allaient attaquer Bougie*. Des contre-attaques hafsides provoquèrent des révoltes contre le makhzen abd-el-wadide jusque dans l'Ouarsenis et la vallée du Chélif'2. C'est alors que le sultan Zianide Hammou Moussa réprima un soulèvement berbère et fonda en 1314 le Ksar Abi Hammou Moussa (aujourd'hui Ammi Moussa).

31 En contant les luttes entre les deux dynasties zianides et mérinides, Ibn Khaldoun parle de l'Ouarsenis comme d'une entité administrative à la tête de laquelle les deux dynasties nommaient gouverneurs des chefs de tribus qui l'habitaient. En 719 H/1319, 
Omar Ibn Othman fut nommé « seigneur du Ouancherich » par Abou Tachfin et lui resta fidèle jusqu'à mourir en repoussant une attaque mérinide contre Tlemcen. Peu après 736 H/1336, le mérinide Abou l'Hacen, qui assiégeait Tlemcen abdelouadide, envoya une armée soumettre tout le pays jusqu'à Médéa inclusivement. "Il donna le gouvernement du Ouancherîch à Nasr, fils d'Ibn Omar Ibn Othman (chef des BeniTîgherîn). Celui-ci se montra bon administrateur et sincèrement dévoué aux Mérinides, consacrant tous ses soins à développer la prospérité de sa province... » (Ibn Khaldoun, t. IV, p. 17, cf. ibid, p. 221).

La domination des Mérinides installés à Tlemcen à partir de 1337 favorisa l'installation des arabes hilaliens (Ouled Ryah) au nord de Chlef, au contact de l'Ouarsenis. Dans les parties basses et plates, les deux populations fusionnèrent sur la base de l'identité du mode de vie nomade, et à l'avantage de la langue arabe. Soustraits au contraire à cette "bédouinisation", les montagnards sédentaires et berbérophones continuèrent d'abord leur existence de planteurs d'arbres et de modestes laboureurs. Ce n'est que plus tard et très progressivement que le massif lui-même reçut des apports de populations arabophones, et que progressa l'arabisation sur place (et parfois récente) des populations berbères, ce qui suffit à expliquer la persistance de coutumes et d'une toponymie essentiellement berbères en pays maintenant majoritairement arabophone.

On peut se demander quel a été l'impact réel de ces dominations successives sur l'intérieur du massif, au-delà de la levée périodique des impôts. C'est à peine si quelques épisodes présentent un caractère un peu différent, avec, par exemple, l'instauration d'une principauté indépendante, mais elle fut vite soumise par les Zianides. Cependant, contrairement aux autres conquérants, ces derniers semblent avoir eu une véritable influence sur l'intérieur du pays, influence attestée par la toponymie, notamment: le Ksar Hammou Moussa (Ammi Moussa) déjà cité, le djebel défensif de "Sidi Hammou» situé entre les territoires des communes de Sebt et Malaab, point de contrôle des accès de la partie occidentale de l'Ouarsenis, le lieu-dit «Khandaq Youcef » dans la commune de Sebt, lieu de la bataille livrée par Youcef Ibn Abou Hamou Moussa en l'an 777 H/1375 et le lieudit "Kherbet Ezziayna" dans la commune de Meghila qui recèle des vestiges datant, dit-on, des Zianides comme semble l'indique le toponyme.

Après 1511, la région suivit le sort des Zianides qui se soumirent d'abord aux Espagnols d'Oran, puis succombèrent en 1555 devant les Turcs.

Quelques années plus tôt, vers 1530, Jean-Léon l'Africain décrit le massif en quelques lignes (Description, 1980, p. 355):

«GUANSERIS montagne. C'est un mont très élevé, habité par une noble tribu qui a fait plusieurs fois la guerre aux rois de Tlemcen. Grâce à l'appui des rois de Fez, ces guerres ont duré plus de soixante ans et davantage. Ce mont a un sol excellent pour la culture et il abonde en sources. À son sommet, qui est très aride, on trouve une grande quantité de tutie [oxyde de zinc, minerai de zinc]. Il fait environ vingt mille combattants dont les deux mille cinq cents cavaliers. Les habitants de cette montagne sont ceux qui apportèrent leur aide au prince Iahia, celui qui lut fait roi de Tenez. Mais depuis que l'état de Tenez a changé de seigneurie, les chevaliers de cette montagne se sont mis à faire des incursions dans le pays ».

On a ainsi trace pour la première fois d'une véritable personnalité politico-militaire du massif, mais la situation n'était sans doute pas nouvelle.

La montagne retourna ensuite au silence des sources historiques jusqu'à la conquête française. 


\section{La période coloniale} massif. La surexploitation des forêts accéléra l'érosion, le fléau principal - auquel s'ajoute maintenant la pollution - qui remet en cause les modes de vie traditionnels (Sari 1977a). différentes parties à cinq wilayates dont les chefs-lieux sont installés à la périphérie : Aïn Defla, Chlef, Relizane, Tissemsilt, Tiaret. C'est vers eux que sont dirigées les routes nouvelles qui ont désenclavé le massif, ce qui a tendu à une meilleure intégration régionale. L'Ouarsenis, château d'eau des piémonts et des plaines de la région, avec de nombreux barrages, reste aussi un important réservoir de main d'œuvre. limites. L'arabisation, d'origine spontanée et scolaire, a progressé (paradoxalement, les parlers berbères se sont mieux conservés dans le Dahra ou les Traras, pourtant beaucoup plus accessibles). 

jaune sous la résine qui sert de vernis) et décor géométrique rouge, mériterait d'être plus étudiée et mieux mise en valeur.

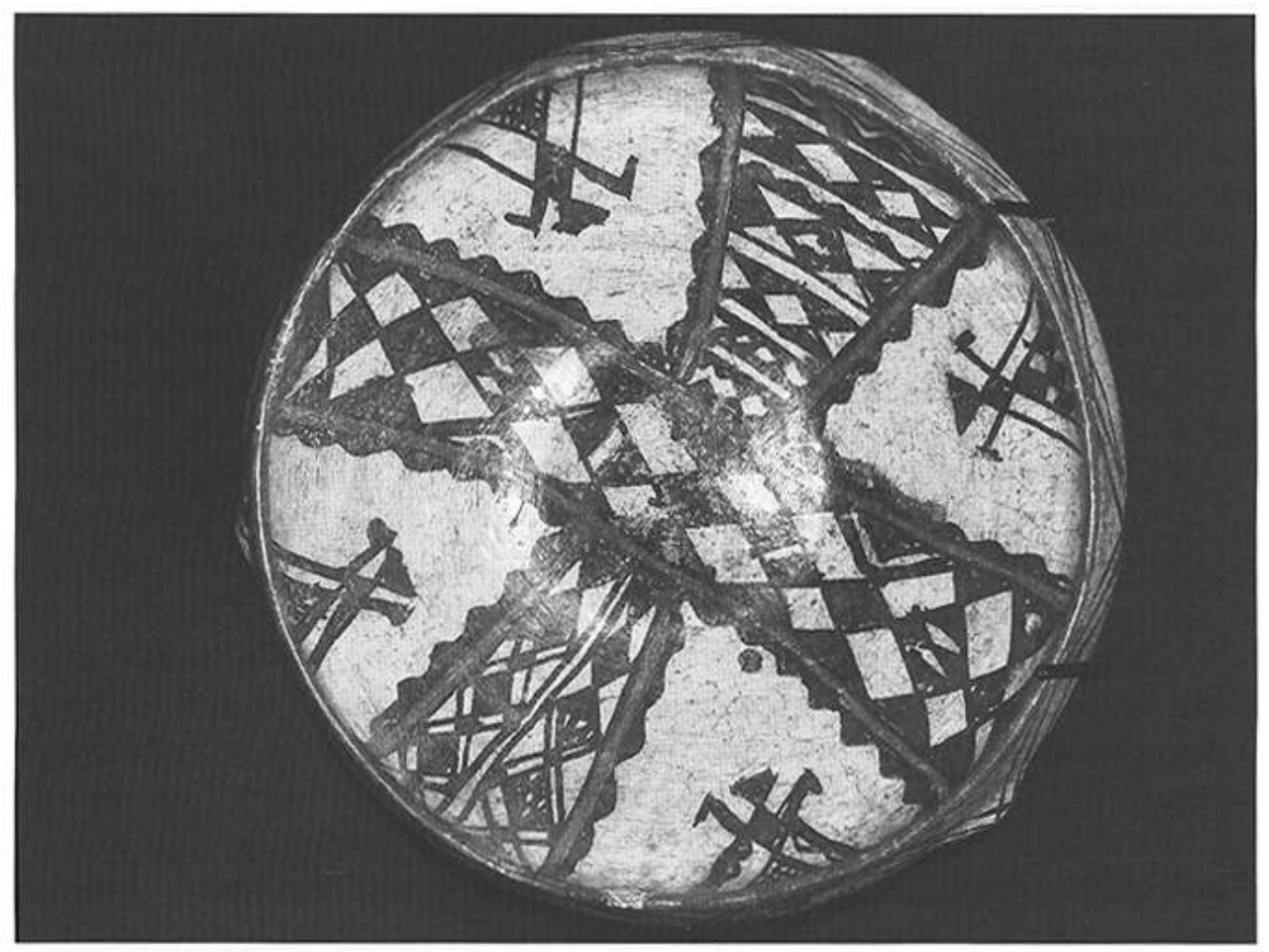

Fig. 2 : PLAT dONNÉ (ON NE SAIT PAS À QUI) EN 1917 (SELON UNE ÉTIQUETTE ANCIENNE COLLÉE SUR LE FOND), PAR UN CHEF DE FRACTION D'AIIN SOLTAN, DU DOUAR DES BENI MERZOUg. COLLECTION E. HAMEL (PARIS)

Cliché P. Richard.

Les troubles islamistes de la décennie 1990 ont de nouveau contraint beaucoup de villageois à abandonner le massif pour se réfugier dans les villes voisines. Dans le même temps, des milliers d'hectares de forêt ont été incendiés.

l'Ouarsenis une partie de ses paysages d'antan. Les richesses naturelles du massif restent considérables et pourront, lorsque les conditions seront redevenues plus favorables, constituer un attrait touristique important.

\section{Société et culture traditionnelles}

En matière d'ethnographie traditionnelle, il reste beaucoup à relever sur les objets et les coutumes, du moins ce qui peut en subsister après tant de bouleversements, pendant plusieurs générations successives.

Fort diminuée aujourd'hui, la poterie traditionnelle à fond blanc (prenant un aspect

Parmi les coutumes locales on signalera un dispositif funéraire inhabituel avec des stèles en bois d'un anthropomorphisme parfois très accentué à Sidi Amara (près d'Ammi Moussa), sur lesquelles F. E. Roubet a recueilli une documentation précise et bien localisée (notamment Roubet 1947). J. Vignet-Zunz en a vu également à Beni Indel, Ouled Ghalia, et leur emploi lui semble général. G. Camps (1962) a montré que cette pratique n'est pas isolée et dépasse largement l'Ouarsenis (Camps 1962) - elle se retrouve dans plusieurs régions de l'ouest algérien, notamment dans les monts des 
Beni-Chougran/Bou-Hanifia. On consultera à ce sujet la notice "Stèles » (à paraître). Comme l'a souligné G. Camps, il faut se garder d'y voir un trait purement berbère, mais ces objets se rattachent certainement à des usages anciens.

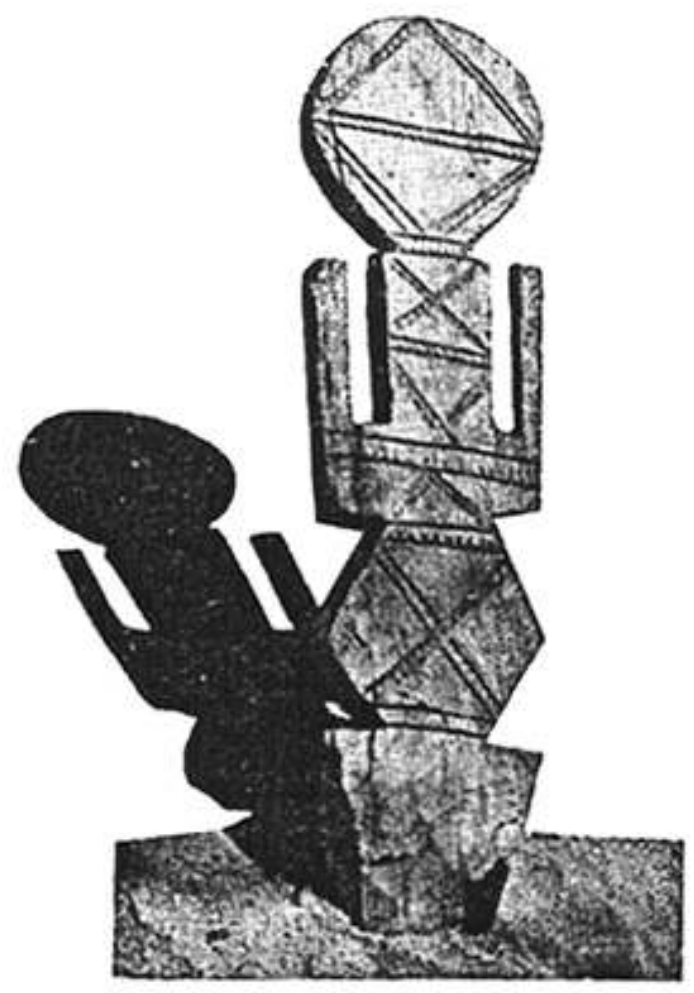

Fig. 3 : STÈLE ANTHROPOMORPHE DE SIDI AMARA PRÈS D'AMMI MOUSSA D'APRÈs F.-E. ROUBET, BSGAO, T. 68, 1947, P. 91 (fig. 1).

\section{Population et organisation sociale traditionnelles}

La géographie humaine de l'Ouarsenis des deux derniers siècles est traitée dans une série d'articles du géographe $\mathrm{Dj}$. Sari dans les années 1970. On pourra également consulter utilement les travaux de J. Vignet-Zunz pour les décennies 1960 et 1970. Bien au-delà de l'ethnographie classique, ces travaux géographiques et sociologiques éclairent le fonctionnement et la désagrégation partielle d'une société montagnarde qui avait construit un équilibre avec la nature, mais qui a subi les coups de boutoir de la colonisation, de la guerre d'indépendance, puis des maquis islamistes, accentués maintenant par les fléaux modernes que sont la surexploitation des forêts, l'érosion et la pollution.

\section{La répartition de la population}

La répartition de la population présente une particularité étonnante: la densité humaine est d'autant plus forte que l'altitude est élevée, et ce trait paraît ancien.

Les zones élevées étant peu productives, l'augmentation rapide de la population (malgré l'exode rural) dans le dernier demi-siècle a été difficilement supportée et a constitué l'une des principales causes des dégradations environnementales actuelles. Ceci n'était pas le cas jadis, grâce à une organisation sociale bien adaptée. 


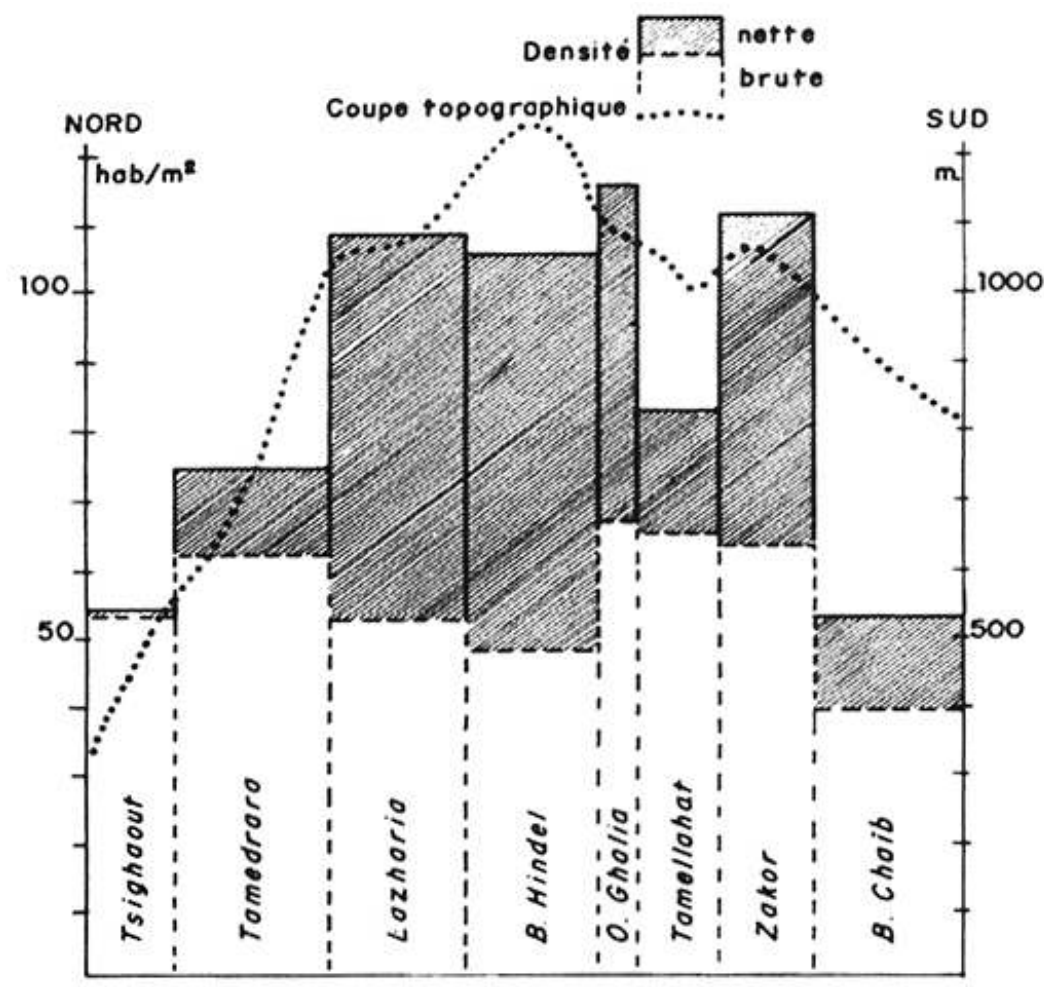

Fig. 4 : RÉPARtition de LA POPULATION de L'OUARSENIS En FONCtion des ALtitUdes. (D'APRÈs DJ. SARI 1972, P. 95.)

\section{La structure sociale}

La structure sociale ancienne comportait trois niveaux : la famille, la buqza (Sari écrit bocca et Vignet-Zunz boq'a ; cf. note 3) et la tribu.

\section{La famille}

La vie familiale s'appuyait sur des ressources diversifiées : cultures vivrières, céréales, produits de cueillette. En hiver, elle s'abritait dans une maison en dur, avec une solide charpente en bois de thuya, recouverte d'une épaisse couche de diss imperméable, et résistant au poids de la neige. Dès le retour des beaux jours, en général vers la mi-avril, jusqu'aux labours et aux cueillettes d'automne, la famille quittait la mechta et partait avec le bétail, soit vers les basses terrasses, soit vers les parcelles éloignées, pour différents travaux, en vivant souvent sous la tente. Ces mouvements saisonniers, qui ne touchaient qu'une partie de la population, prirent fin à la fin du XIX ${ }^{e}$ siècle suite à la désorganisation des terroirs (Sari 1971, p. 86).

L'humidité de la montagne est suffisante pour l'arrosage des jardins, les moissons et les récoltes et permet des pâturages réguliers, contrairement à ce qui se passe dans les plaines. La prédominance des cultures irriguées et en particulier de l'arboriculture s'expliquait non seulement par des facteurs physiques (ressources hydrauliques importantes, exposition, fixation des pentes et terrasses) mais aussi par des raisons juridiques : la possession privée et directe de la terre (maintenant fort diminuée ; cf. Sari 1971). Avant les diverses spoliations de la période coloniale (Sari 1975), la propriété privée des terres remontait loin, avec des actes datant des XVI ${ }^{e}$ et XVII ${ }^{e}$ siècles. Il ne 
peut s'agir de populations errantes et de populations réfugiées mais bien, très anciennement, d'une population sédentaire avec des droits bien définis (cf. note 4). Du côté de l'arboriculture, les essences les plus courantes sont les figuiers, qui occupent le plus d'espace, puis les oliviers, le grenadier, le prunier et l'amandier, et partout le chêne vert à gland doux ${ }^{3}$ (fourrage pour les animaux). Même si le nombre d'animaux par $\mathrm{km}^{2}$ était plus faible que dans la plaine du Chélif, il était relativement élevé sur des surfaces limitées, notamment dans les bas-versants et les plaines les plus favorables. Les activités complémentaires, artisanat, mais aussi location des bras dans les plaines étaient nécessaires à l'équilibre économique des différentes tribus.

Au sein de la buqza, et en dehors des travaux à caractère collectif, chaque famille élargie (ménage comprenant le plus souvent les grands parents, les parents, et les enfants) vaquait librement à ses occupations, entretenant les parcelles irriguées, ou s'attelant à d'autres tâches, puisque, comme on l'a déjà précisé, la propriété était essentiellement privée ; cela n'était pas incompatible avec les besoins de la buqza (Sari 1971, p. 86).

Depuis des temps immémoriaux, les différentes fractions semi-nomades du sud avaient des droits d'usage sur les pentes méridionales de l'Ouarsenis. Ces liens économiques furent renforcés par des liens matrimoniaux. On note une complémentarité de même type économique sur le versant septentrional, en direction de la vallée du Chélif.

\section{La buqea}

La buqza apparaît comme une structure socio-économique originale, intermédiaire entre la famille et la tribu. Tant Dj. Sari que J. Vignet-Zunz ont souligné son importance dans l'équilibre de jadis entre la population et son environnement. Ce mode d'organisation débordait à la fois sur les plaines du Chélif, la chaîne du Dahra et sur les hautes-plaines du Sersou.

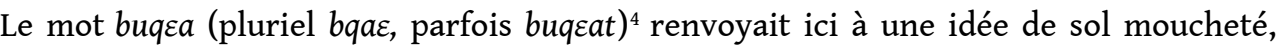
alternativement clair et sombre, sec et vert. Il s'appliquait bien au Tell quand ses pentes, plus boisées qu'aujourd'hui, étaient coupées de clairières, chacune occupée par l'habitat hivernal d'une buqea.

60 L'habitat dispersé de l'Ouarsenis se répartissait en une infinité de petites communautés liées chacune à un territoire comportant des zones arboricoles et céréalières et conçues comme autant d'unités généalogiques (réelles ou conventionnelles). La buqعa apparaît à la fois comme une communauté lignagère et comme une entité socio-économique particulière, qui unit plusieurs types d'organisation, sociale, économique, territoriale et agricole. Le mot qualifiait en fait à la fois l'organisation d'un territoire et celle de ses habitants.

61 En schématisant quelque peu, la partie centrale de la buqea, la mechta, résidence d'hiver, était constituée par un hameau d'une dizaine de maisons entourées de jardins, formant une clairière bien visible de loin sur fond de forêts. Son territoire comprenait également plus haut des zones forestières, et plus bas, sur le piémont voisin, des champs cultivés (souvent en céréales). Dès le retour des beaux jours, la population (aujourd'hui entièrement sédentarisée) se déplaçait vers ses autres terroirs en logeant sous la tente ou dans de modestes habitations de pierre couvertes de diss (jonc séché).

Tous les membres de la buqza étaient solidaires et concernés par tout ce qui touchait à l'organisation de leur espace et à leur existence même. La buqza organisait notamment les travaux collectifs : construction de séguias, de pistes, de barrages de dérivation, 
d'aménagement de sources, ou d'autres travaux exigeant le concours de la collectivité, il fallait faire appel à toutes les personnes valides. Ces travaux se déroulaient alors dans le cadre de la "touiza" [twiza $]^{5}$ ou "travaux collectifs d'entraide". Sur le plan culturel, la buqza était responsable de l'enseignement primaire des enfants et de la célébration de certaines fêtes à caractère local. En général, tous les enfants masculins, mais rarement les filles, allaient à l'école de la buqza, pour apprendre par coeur les versets du Coran et les rudiments de la langue arabe. Le maître était entretenu par les volontaires seulement. Pour un enseignement plus complet, il fallait fréquenter les établissements scolaires de la vallée du Chélif, conservés encore en partie jusqu'à la veille de l'indépendance (Sari 1971, p. 86-87).

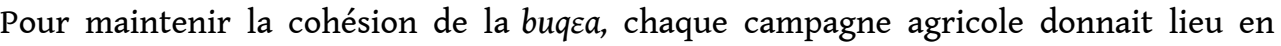
général à une cérémonie que tout le monde devait respecter. Celle des labours était toujours inaugurée par un personnage reconnu de tous. C'est seulement après le coup d'envoi donné par ce personnage incontesté que les travaux démarraient.

Chaque buqsa devait s'acquitter annuellement d'une dette envers son wali ou saint, c'est-à-dire en définitive l'ancêtre commun, personnage qui s'était distingué par ses talents, sa foi, sa vaillance... Aussi continuait-on à le vénérer, en offrant toujours à la même date, le țam, des offrandes alimentaires au profit de tous les présents et participants, afin que le TrèsHaut les bénisse et admette leur âme parmi les élus.

Lorsque le cadre de la buqga devenait trop étroit, il fallait alors agir dans un autre plus grand, celui de la tribu. Plus que le maire qui est à l'heure actuelle très peu en rapport avec ses électeurs, à la suite du regroupement des communes (depuis 1964), c'est le

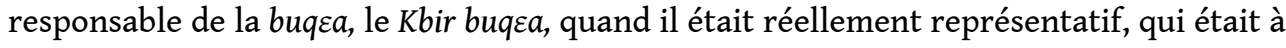
même de jouer un rôle positif, en défendant bien les intérêts de sa buqza, au niveau de la tribu (Sari 1971, p. 87, n. 28). C'était plus un animateur et un conciliateur qu'un chef.

\section{La tribu}

La tribu se composait d'une dizaine de buqzat (de six à douze), gérant les conflits entre elles et défendant leurs intérêts vis-à-vis de l'extérieur. En principe ignorée, depuis longtemps, des structures administratives modernes, cette organisation traditionnelle sous-jacente, semble encore s'exprimer de temps à autre par des voies diverses qui mériteraient d'être explorées.

\section{BIBLIOGRAPHIE}

BAYLE DES HERMENS R., 1955 - « Les gravures rupestres de l'oued Seffalou. Région de Tiaret.

Département d'Oran », Libyca, III, Alger, p. 327-343.

BAYLE DES HERMENS R., 1956 - « Station de gravures rupestres d'Aïn-Sfa. Région de Vialar », Libyca, IV, p. 135 
BAYLE DES HERMENS R., 1963 - «Influences sahariennes dans le néolithique de la région de Tiaret (Algérie) », Bulletin de la Société préhistorique française, vol. 60, n 1-2, p. 79-91.

BERBRUGGER A., 1856/1857 - « Antiquités du cercle de Ténès », Revue africaine, vol. 5, p. 335-345 \& vol. 7, p. 182-83.

BOUDIBA M., 2003 - L'Ouarsenis. La guerre au pays des cèdres, Paris, L'Harmattan.

CADENAT P., 1963 - « L'art rupestre préhistorique du département de Tiaret (Algérie)», Soc.

d'Etudes et de recherches préhistorique (Les Eyzies, Institut pratique de Préhistoire, Bull. $\mathrm{n}^{\circ} 13$, trav. de 1963), p. 34-51.

CAMPS G., 1962 - « Remarques sur les stèles anthropomorphes en bois de l'Afrique du Nord », Libyca, 9-10, 1961-1962, p. 205-221.

CAMPS G., 1995 - Notice D63. « Djedar », EB XVI, p. 2409-2422.

CASTELLANi M., JOLEAUD L., 1935 - « Escargotière préhistorique de Champlain, près de Médéa (Alger) », Journal de la Société des Africanistes, vol. 5, n 5-2, p. 159-162. [L. Balout signale la présence de l'Homme de « Mechta-Afalou* » dans cette escargotière dite du « Tléta » au sud-ouest de Champlain [= El-Omaria, w. de Médéa], découverte à l'est du marabout : L. Balout, Préhistoire de l'Afrique du Nord..., Paris, AMG, 1955, p. 232].

CÔTE M., 2005 - « Wanshari(s), Ouarsenis », Encyclopédie de l'Islam, t. X, V-Z, p. 152-153.

COURTOIs Chr., Les Vandales et l'Afrique, Paris, AMG, 1955, 457 p.

DESANGES J., 1962 - Catalogue des tribus africaines de l'Antiquité classique à l'ouest du Nil, Dakar, Publications de la section d'Histoire de l'Université de Dakar, nº 4, 1962, 296 p., 10 cartes.

DESANGES J., 2010 - s.v. « Makourae », EB, XXX, p. 4535

IBN KHALDOUN, Histoire des Berbères, 4 vol., Paris, 1925 (rééd.).

JULIEN Ch.-A., 1951/1978 (nombreuses rééditions) - Histoire de l'Afrique du Nord, Payot, 2 t.

LAPORTE J.-P., 2003 - «Ptolémée et la Maurétanie césarienne », CRAI, 2003, p. 171-195.

LAPORTE J.-P., 2004 - « Les armées romaines et la révolte de Firmus (370-373), Congrès L'Armée romaine de Dioclétien à Valentinien Ier, Lyon, 2002 (2004), p. 269-288.

LAPORTE J.-P., (à paraître) - « Ksar el-Kaoua (Ammi Moussa, w. Chlef) : une maison-forte en Maurétanie césarienne", Colloque de Rouen, 2009.

LÉON L'AFRICAIN Jean-Léon, Description de l'Afrique, éd. A. Epaulard, Paris, Maisonneuve et Larose, 1980, 2 vol.

MODERAN Y., Les Maures et l'Afrique romaine (IV ${ }^{e}$-VII ${ }^{e}$ siècle), Collection de l'EFR, BEFAR 314, 2003, $900 \mathrm{p}$.

REBUFFAT R., 2007 - « Makkoioi, Les peuples de Maurétanie césarienne occidentale », colloque L'onomastique africaine, SEMPAM et Université de Sassari (sous presse).

ROUBET F.-E., 1947a - « Le combat de buffles de l'Oued Azouania », Bull. de la Soc. de Géographie et d'Archéologie d'Oran, t. L. XIX, p. 62-63.

ROUBET F.-E., 1947b - « Etude sur la stylisation des stèles anthropomorphes de l'Ouarsenis », Bull. de la Soc. de Géographie et d'Archéologie d'Oran, t. 68, p. 83-95.

SALAMA P., 1954 - « Inscription maurétanienne de 346 », Libyca, II, 1,1954, p. 229. 
SALAMA P., 1966 - « Occupation de la Maurétanie occidentale sous le Bas-Empire romain », Mélanges Piganiol, Paris, 1966, p. 1291-1311 = Promenades d'Antiquités africaines, 2005, p. 169-189.

SARI Dj., 1969 - « L'activité minière de l'Ouarsenis », Annales algériennes de Géographie, 7, 1969, p. 7-25.

SARI Dj., 1971 - « L'équilibre économique traditionnel des populations de l'Ouarsenis central », ROMM, 9, p. 63-89

SARI Dj., 1972 - «Les populations de l'Ouarsenis central », Méditerranée, (2érie), 11/3-4, p. 89-117.

SARI Dj., 1975 - La dépossession des fellahs 1830-1962, Alger, SNED, 1975

SARI Dj., 1977a - L'homme et l'érosion dans l'Ouarsenis, Algérie, Alger, SNED, 1977, 623 p.

SARI Dj., 1977b - « Ouarsenis », Techniques et cultures (Bulletin de l'équipe de recherches 191, CNRS), 2.

SARI Dj., 1978 - « Les problèmes de l'environnement dans les pays sous-développés, le déboisement dans l'Ouarsenis ", in Nesson Cl., Sari Dj., Peillon P., Recherches sur l'Algérie, Paris, Éditions du CNRS, 291 p.

SARI Dj., 1979 - « Les tentatives de restructuration du monde rural en Algérie », Méditerranée, $3^{\text {e }}$ sér., t. 35, 1-2, (L'homme et son milieu naturel au Maghreb), p. 65-72.

SUDRY Y., 2008 - Guerre d'Algérie : les oueds rouges de l'Ouarsenis, Paris, L'Harmattan.

VIGNET-ZUNZ J., 1972 - Homme de l'Ouarsenis : une communauté rurale d'Algérie, Thèse de Doctorat de $3^{\mathrm{e}}$ cycle, Paris, Université René-Descartes (M. Leroi-Gourhan, Dir.). Disponible sur microfiches : 1974, Paris, Institut d'Ethnologie (Archives et Documents), microfiche $\mathrm{n}^{\circ}$ 74.012.103. Cf. notamment Chap. II/6, «L'Ouarsenis : sa personnalité et l'hypothèse berbériste ».

VIGNET-ZUNZ J., 1977 - « L'habitat rural dans l'Ouarsenis (Algérie) », Techniques et Cultures, 2, p. 27-78.

VIGNET-ZUNZ J., 2012 - Montagnes et savoirs en Méditerranée. Ouarsenis (Algérie), Jabal Al-Akhdar (Libye), Rif(Maroc), Recueil d'articles, Alger, CNRPAH (\& Aix-en-Provence, IREMAM).

YACONO X., 1955 - La colonisation des plaines du Cheliff..., Alger, Imp. Imbert, vol. I, 445 p.

\section{NOTES}

1. CIL, VIII, 21531 et 21532 à Ammi Moussa ; Libyca, II, 1954, p. 206-207 (Kherba des Aouissat), BCTH, 1910, p. CLXXIX (Damouni) ; Libyca, ibid., p. 220 (Aïn Sidi el Hadj) ; CIL, VIII, 9613 (Miliana) ; etc.

2. Ibn Khaldoun, Histoire des Berbères, t. III, 350, 392-395, t. IV, 207. Yahya Ibn Khaldoun, Beni Abd el Wad, I, p. 173-175. Brunschwig, Hafsides, I, p. 132 et n. 5.

3. Il s'agit du « chêne ballote» (Quercus ilex subsp. ballota (Desf.) Samp.), dont les glands doux pouvaient autrefois être consommés par les humains par temps de disette - ce fut encore le cas dans bien des régions d'Algérie pendant la Seconde Guerre mondiale. [S.C.].

4. Le terme buqza est attesté à la fois en arabe classique, où il signifie « contrée, lieu, endroit », et en arabe dialectal maghrébin: en Algérie, il signifie «endroit, lieu, champ, pièce de terre, terrain; sol, pays; sous-fraction de tribu» (M. Beaussier, Dictionnaire pratique arabe [dialectal algérien]-français, Alger, 1887/1931, p. 66); au Maroc, sous la forme beqદa, "pièce de terre, 
parcelle de terre non bâtie, terrain, territoire » (A.-L. de Prémare, Dictionnaire arabe-français, t. 1, Paris, L'Harmattan, 1993, p. 279). La signification fondamentale de ce mot est donc manifestement liée au sol et au territoire, et non à l'organisation sociale/tribale, sens qui paraît nettement secondaire. Il semble donc que la notion locale de buqعa relève de la réintégration d'une réalité territoriale/agricole dans un tissu tribal. [S.C.].

5. Twiza est la forme arabisée du mot berbère tiwizi, «travail d'entraide collectif », qui, dans la société traditionnelle était une obligation qui s'imposait à tous les membres du groupe (clan, village, voire tribu), pour tous les travaux, saisonniers ou non, exigeant une grosse mobilisation de main-d'œuvre: cueillettes et récoltes diverses, constructions individuelles ou collectives, travaux d'intérêt général... [S.C.].

INDEX

Mots-clés : Algérie, Antiquité, Ethnologie, Géographie, Maurétanie, Moyen Âge 